\title{
Effects of Autonomic Nervous System Activation on Endothelial Function in Response to Acute Exercise in Hypertensive Individuals: Study Protocol for A Randomized Double-Blind Study
}

\section{Gustavo Waclawovsky}

Institute of Cardiology of Rio Grande do Sul/University Foundation of Cardiology

Liliana FC Boll

Institute of Cardiology of Rio Grande do Sul/University Foundation of Cardiology

Salvador Gomes Neto

Institute of Cardiology of Rio Grande do Sul/University Foundation of Cardiology

Maria Cláudia Irigoyen

Institute of Cardiology of Rio Grande do Sul/University Foundation of Cardiology

Alexandre Machado Lehnen ( $\nabla$ amlehnen@gmail.com )

Instituto de Cardiologia do RS https://orcid.org/0000-0002-5912-8020

\section{Study protocol}

Keywords: endothelium, autonomic nervous system, exercise, systemic arterial hypertension

Posted Date: September 21st, 2020

DOI: https://doi.org/10.21203/rs.3.rs-32356/v1

License: (c) (1) This work is licensed under a Creative Commons Attribution 4.0 International License. Read Full License

Version of Record: A version of this preprint was published at Trials on August 19th, 2021. See the published version at https://doi.org/10.1186/s13063-021-05516-X. 


\section{Abstract}

Background: Arterial hypertension has a direct association with endothelial dysfunction and major cardiovascular events. There is evidence showing the benefits of aerobic exercise on flow-mediated dilation (FMD) in hypertensive individuals but little is known about the effect of autonomic nervous system (ANS) activation on FMD of the brachial artery in response to different types of exercise in this specific population. This study aims to examine the effects of ANS activation on FMD of the brachial artery in response to exercise in hypertensive individuals following a session of different types of exercise including aerobic exercise (AE), resistance exercise (RE) or combined exercise (CE).

Methods: Thirty-nine hypertensive volunteers aged 35 to 55 years will be randomly assigned to two exercise sessions: AE (40 minutes on a cycle ergometer at $60 \%$ of HR reserve); RE (4 lower limb sets with 12 repetitions at $60 \% 1-\mathrm{RM}$ for 40 minutes) or CE (RE for 20 minutes $+\mathrm{AE}$ for 20 minutes). Each exercise group will be randomized to receive either an a1-adrenergic blocker (doxazosin $0.05 \mathrm{mg} / \mathrm{kg}-1$ ) or placebo. Ultrasound measurement of FMD is performed 10 minutes before and 10, 40 and 70 minutes after exercise. ANS activation is monitored using a Finometer and measurements are taken during 10 minutes before each FMD assessment. Arterial stiffness is assessed by pulse wave velocity (PWV) analysis using a Complior device.

Discussion: We expect to demonstrate the effect of ANS activation on FMD of the brachial artery in hypertensive individuals in response to different types of exercise. This study may give some insight on how to improve exercise prescription for hypertension management.

Trial registration: www.clinicaltrials.gov (ID: NCT04371757).

\section{Introduction}

Endothelial dysfunction precedes atherosclerosis (1). Endothelial cells play an important role in modulating vascular angiogenesis and vascular tone and permeability and mediating inflammatory responses and homeostasis (2). This function of vascular protection is largely attributed to nitric oxide endothelial synthase, an enzyme responsible for nitric oxide (NO) synthesis in the vascular endothelium (2).

Endothelial dysfunction is characterized by a reduction of local NO bioavailability and/or insufficient vasomotor response that occur in certain conditions including arterial hypertension (3), diabetes mellitus (4), obesity (5), dyslipidemia (6), metabolic syndrome (7), aging (8), menopause (9) and sedentary lifestyle (10). Flow-mediated dilation (FMD) of the brachial artery is an indirect non-invasive technique to assess endothelial function and is chiefly dependent on NO availability (11). Since acute post-exercise changes in vascular function may also predict long-term adaptations (12) the acute response to exercise has been widely studied $(3,13)$. 
Lifestyle changes including regular physical exercise is recommended as part of the therapeutic approach for hypertension management $(14,15)$. Factors most commonly associated with potential antihypertensive effects and chronic post-exercise changes in endothelial function include reduced autonomic nervous system (ANS) activation (16), balance between vasodilators and vasoconstrictors (17) and reduced levels of the vasoconstrictor endothelin-1 (ET-1) (18).

A meta-analysis that examined cardiovascular risk factors and/or factors associated to established cardiovascular disease found that aerobic and resistance training can potentially improve FMD of the brachial artery (19). Our research group also found an improvement of $1.45 \%(95 \% \mathrm{Cl}-0.11$ to 3.00$)$ in FMD in response to aerobic training in hypertensive individuals (20). This finding is clinically relevant as an $1 \%$ increase in FMD is correlated to $~ 13 \%$ risk reduction of major cardiovascular outcomes (21).

A study of the effects of resistance exercise (RE) on FMD (22) found that a single session of low- and moderate-intensity knee extensions reduced FMD 10,30 and 60 minutes after exercise in healthy individuals. Interestingly, FMD did not change when this same set of RE was performed at high intensity (22), which may indicate an inverse relationship between FMD changes in response to RE as well as to exercise intensity. Our research group have recently reported that a single session of aerobic exercise $(\mathrm{AE})$, lower-limb $\mathrm{RE}$ and $\mathrm{CE}(\mathrm{AE}+\mathrm{RE})$ at moderate intensity for 40 minutes did not result in brachial artery FMD changes in hypertensive individuals when measured 10, 40 and 70 minutes after exercise (23). In addition, the levels of endothelial microvesicles and substances associated with oxidative stress remained unchanged (23). Altogether, we can raise the hypothesis that other factors may play a role including exacerbated sympathetic activation in untrained limbs, which may cause vasoconstriction and redirection of blood flow to trained limbs $(24,25)$, thus potentially competing with endothelium-dependent vasodilator effect (26).

Alves et al. (27) demonstrated that a1-adrenergic block with phentolamine reduced blood flow in the forearm when compared to placebo during a handgrip exercise session in individuals with heart failure. It has been proposed that exacerbated sympathetic activation plays an important part in the development and maintenance of arterial hypertension (28), so we postulate that following an acute exercise session sympathetic activation potentiates vasoconstriction and may compete with endothelium-dependent vasodilation effects in hypertensive individuals.

In view of inconsistent findings of the acute effects of different types of exercise on FMD in healthy and hypertensive individuals as well as the scarcity of data of the effects of ANS activation on vascular reactivity in response to exercise in hypertensive individuals, we designed a randomized clinical trial with the primary objective of examining the effects of ANS activation on FMD of the brachial artery in response to acute exercise ( $\mathrm{AE}$, lower-limb RE and $\mathrm{CE}$ ) in hypertensive individuals. Table 1 summarizes the study objectives. 
Table 1

- Study objectives

\section{Primary objective}

- To compare measurements of FMD of the brachial artery in hypertensive individuals receiving either an a1-adrenergic blocker or placebo after sessions of different types of exercise.

\section{Secondary objectives}

- To compare the effects of ANS activation in hypertensive individuals receiving either an a1adrenergic blocker or placebo (between-group) in response to acute exercise (aerobic, resistance and combined exercise sessions);

- To correlate the levels of arterial stiffness and ANS activation and FMD of the brachial artery in hypertensive individuals receiving either an a1-adrenergic blocker or placebo in response to acute exercise (aerobic, resistance and combined exercise sessions).

FMD: flow-mediated dilation; ANS: autonomic nervous system.

\section{Methods/design}

\section{Study setting}

The study -a parallel-group randomized, double-blind (placebo/drug but not to exercise type) clinical trial -is approved by the local ethics committee (\#5678/19) and it will be conducted at Instituto de Cardiologia do Rio Grande do Sul/Fundação Universitária de Cardiologia (study site). All eligible individuals must read and sign a free and informed consent (supplementary material) before entering the study. The study protocol is being developed following the principles of the Declaration of Helsinki. All study information is confidential; volunteers' names will be kept confidential and all data obtained in the study will be used for academic purposes only. Research data will be stored for five years and then discarded according to the guidelines of CNS Resolution No. 466/12. Finaly, any adverse effect during the research will be reported to the local ethics committee that will have access to the adverse results and will make the decision in relation to terminating the study, if aplicable.

\section{Trial Status}

The study is registered on "www.clinicaltrials.gov" (ID: NCT04371757), as follows: Last update posted on May 1, 2020; Estimated study start on April 30, 2020; Estimated primary completation on December 20, 2020; Estimated study completation on December 23, 2021. At moment the recruitment status is

"Recruiting".

\section{Eligibility criteria}

Recruitment will be through on-site screening, media postings, and patient databases. Thus, hypertensive male and female individuals aged 35 to 55 years receiving health care at the study site, using antihypertensive drugs, who do not engage in regular physical exercise ( $\geq 2$ sessions per week) are eligible to 
participate. Exclusion criteria are individuals with diabetes mellitus; chronic renal failure; body mass index $(\mathrm{BMI}) \geq 35 \mathrm{~kg} / \mathrm{m}^{2}$; coronary artery disease; heart failure; users of beta-blockers and/or alpha-blockers; smoking; lower limb injuries that prevent them from engaging in the study intervention; and females aged 35 to 55 with menopause.

\section{Sample size}

The sample size is calculated according to Atkinson et al. (26). In their study, they compared endothelium-dependent vasodilation (by FMD) after a single session of moderate-intensity AE for 30 minutes in healthy individuals receiving an a1- adrenergic blocker (prazosin $0.05 \mathrm{mg} / \mathrm{kg}^{-1}$ ) or placebo and reported a difference in means of $3.1 \%$ with standard deviations (SDs) of $1.13 \%$ for placebo and $1.91 \%$ for prazosin. For finding a similar difference in FMD (3.1\%) between these two conditions (a1-adrenergic receptor block versus placebo) after our protocol's exercise session (AE, RE and $C E$ ) considering $a=0.05$ and $80 \%$ power, a sample of five volunteers per group $(n=15)$ is required.

For a comparison of FMD measurements among exercise types (AE, RE and $C E$ ), our sample calculation is based on pre-exercise SD (1.18\%) and post-exercise SD (1.91\%) for the prazosin group as described by Atkinson et al. Thus, to find a difference in FMD of $1.62 \%$ considering $a=0.05$ and $80 \%$ power, a sample of 10 individuals per group $(n=30)$ is required. Assuming a $30 \%$ loss, a sample of 39 individuals would be required ( $n=13$ per group).

\section{Randomization and blinding}

Randomization of the study interventions (exercise groups) as well as assignment to intervention conditions (either an a1- adrenergic blocker or placebo) are carried out using computer program (www.randomization.com) with a coded numeric distribution. Allocation concealment is guaranteed; the random allocation of volunteers is kept in an inaccessible place and researchers will not have a priori knowledge of the intervention assignment to each volunteer. Randomization is carried out by an independent researcher not involved with the study.

Considering the volunteers' age group (35 to 55 years) and that comparison analyses (primary and secondary outcomes) involve testing for baseline differences, we are conducting simple randomization divided into two steps: (1) by exercise type (AE, RE or CE) and (2) the order of condition (either placebo or a1-adrenergic blocker). All volunteers are blinded to the intervention allocation and will only have access to this information after a baseline evaluation. They are also blinded to the intervention condition (either placebo or a1-adrenergic blockade). The researchers responsible for the analyses of data are blinded to the intervention allocation and condition to minimize potential measurement bias.

\section{Evaluation and interventions}

Volunteers are selected based on information from their medical records. They are first contacted through a telephone call to ascertain whether they fit the inclusion criteria and do not meet any of the exclusion 
criteria. They are then pre-selected and invited to participate in the study. Those who agree to participate are scheduled their first visit (visit 1) at the study site.

Eligible individuals go through a four-step protocol as described below. Figure 1 shows the detailed flowchart of the study; Figure 2 summarizes the recruitment, study interventions and assessments; and Figure 3 describes the study intervention (exercise and condition).

Since the study is designed to assess the acute effects of different types of exercise types under the condition of a1-adrenergic blockade or placebo, female volunteers are to undergo evaluations and exercise sessions during the luteal phase of the menstrual cycle.

\section{Step 1: interview, anthropometric assessment and exercise test}

Visit 1 takes place at the Laboratory of Clinical Investigation (LCl) at the study site. All inclusion and exclusion criteria are ascertained and the principal investigator (GW) will ask volunteers to sign a free and informed consent form. All research data collected will be stored in an appropriate safe space at the study site that is accessible to the principal investigator (GW) only.

They are then administered a medical questionnaire the International Physical Activity Questionnaire long version (IPAQ, http://www.ipaq.ki.se). The volunteer is asked to remain in a seated position in a quiet room for 5 minutes before their blood pressure (BP) is measured (OMRON ${ }^{\text {TM }}$ HEM-714INT device, Tokyo, Japan); three recordings are taken 1 minute apart on the arm with the highest values. Those who meet all inclusion criteria undergo an anthropometry assessment and a pre-scheduled exercise stress test.

The anthropometry assessment takes place at the study site. It includes measurements of total body mass (measured with appropriate one-piece [males] and two-piece clothing [females] in a standing position barefoot with feet together at the center of the scale); height (measured in a standing position with feet together, the head in the Frankfort plane with the stadiometer on the top of the head and readings taken while breathing in); waist circumference (measured in a relaxed standing position with arms folded across the chest with the measuring tape placed around the abdomen at narrowest point between the lower costal margin and the upper part of the iliac crest, the reading is made in the front perpendicular to the longitudinal axis of the body) (29).

The exercise stress test is performed on a treadmill (Inbramed, Porto Alegre, Brazil) using the Bruce Protocol and following the Brazilian Society of Cardiology guidelines (30). In a testing room at the study site a qualified technician and a cardiologist conduct the stress test followed with measurements of maximum oxygen consumption, respiratory parameters and maximum heart rate (HR) (ErgoPC 13, MICRIMED, Brasília, Brazil). Aerobic exercise is to be prescribed according to these measurements. The volunteers are then released by the cardiologist. They are contacted by phone to schedule visit 2 .

\section{Step 2: blood collection, assessment of arterial stiffness and maximum strength test}


On visit 2 the volunteers are asked to come to the study site after a 12-hour overnight fast for blood collection and biochemical analysis (blood count, fasting blood glucose, $\mathrm{HbA1c}$ levels, triglycerides, total cholesterol, low-density lipoprotein cholesterol [LDL-c] and high-density lipoprotein cholesterol [HDL-c], creatinine and ultra-sensitive polymerase chain reaction [PCR] assay). LDL-c levels and glomerular filtration rate (GFR) are calculated as detailed below.

a) $L D L-C=($ total cholesterol $-H D L-C)-($ triglycerides $/ 5)$;

b) $\operatorname{GFR}\left(\mathrm{mL} / \mathrm{min} / 1.73 \mathrm{~m}^{2}\right)=175 \times$ creatinine ${ }^{-1.154} \times$ age $^{-0.203} \times 1.212$ (for blacks) $\times 0.742$ (for females) (31).

Volunteers are then offered a standard snack and asked to rest in a dark, quiet room at $23-24^{\circ} \mathrm{C}$ for 10 minutes. Arterial stiffness is assessed by carotid-femoral pulse wave velocity (PWV) (32) using the Complior Analyse (ALAM Medical, Paris, France). The straight distance between the two arterial sites is measured with an infantometer and data on BP, height, total body mass and distance between carotid and femoral artery sites are entered into the measuring device software for PWV calculation (32).

The volunteers then undergo the one-repetition maximum (1-RM) test to assess lower limb maximum strength (33) in a proper exercise room at the study site. They first learn the exercise techniques and are instructed on how to perform knee extension, knee flexion, leg pressure and plantar flexion exercises on a guided weight machine (Movement Perform W8; São Paulo, Brazil). Warm-up consists of 10-12 repetitions, $30 \%$ of 1-RM (2 concentric: 2 eccentric phases controlled by a metronome). After a rest of 3-5 minutes, they start the 1-RM test. It is interrupted to adjust the load when they are able to perform more than one repetition or when they are not able to lift the load. A 5-minute rest is allowed before a new attempt is made. The 1-RM is determined when the volunteer performs one complete movement (33). Their BP is measured 5, 10 and 15 minutes after the end of the test. The study interventions are scheduled afterwards.

\section{Steps 3 and 4: a1-adrenergic receptor blockage, assessment of endothelial function, autonomic nervous system activation and exercise intervention}

a1-adrenergic receptor blockage: a1-adrenergic blockage is used to assess the effects of sympathetic nervous system (SNS) activation on FMD in response to exercise as described in the literature (26). Since prazosin is no longer available in Brazil, its analogue doxazosin is used in this study (34). Briefly, 90 minutes before the start of the exercise intervention, the volunteers are asked to take a tablet containing either placebo (microcrystalline cellulose) or an a1-adrenergic blocker (doxazosin $0.05 \mathrm{mg}^{\mathrm{kg}}{ }^{-1}$ of body weight) and to lie down and rest. This dose of an a1-adrenergic blocker has been reported as being able to block $80 \%$ of a1-adrenergic receptors in studies of sympathetic activation and vascular responses (3537).

An independent researcher blinded to the study intervention gives a tablet to the volunteer on the day of the exercise session according to previous random assignment. 
Assessment of endothelial function: endothelial function is assessed by FMD as described elsewhere (38, $39)$. In a quiet, dark room with controlled temperature $\left(23-24^{\circ} \mathrm{C}\right)$ at the study site, a qualified evaluator blinded to the intervention condition simultaneously measures blood flow velocity and baseline brachial artery diameter on the volunteer's right arm. With the volunteer in supine position, arm extended and across the trunk at angle of $\sim 40^{\circ}$ after a 10-15-minute rest, measurements of baseline BP (left arm), resting HR, blood flow velocity and brachial artery diameter are taken. They are to be evaluated 10 minutes before and 10, 40 and 70 minutes after the exercise session.

For the assessment of FMD of the brachial artery, the volunteers are asked to come to the study site after 6-hour fasting except for anti-hypertensive medication. A rapid deflation cuff (Incoterm ${ }^{\text {TM }}$ EC500; Porto Alegre, Brazil) is placed around the forearm $5 \mathrm{~cm}$ distal to the antecubital fossa and B-mode artery images are obtained at the distal third of the arm with a linear multi-frequency transducer $(12 \mathrm{MHz})$ connected to a high-resolution Doppler ultrasound system (Esaote MyLab ${ }^{\text {TM }} 70$ XVision; Genoa, Italy). The positions of the ultrasound probe and the cuff are marked on the skin so that they can be reproduced during the study. The position of the arm is photographed and the distance between the transducer and the antecubital fossa is measured so that the same can be reproduced in the second exercise session. The evaluator aligns the cursor of the sample volume to the width of the artery at an insonation angle of $60^{\circ}$. Baseline diameters are recorded for 1 minute and the forearm cuff is inflated to $50 \mathrm{mmHg}$ above systolic blood pressure (SBP) for 5 minutes. Images are recorded 30 seconds before cuff deflation and for 3 minutes after cuff deflation. Real-time Doppler ultrasound images are recorded and stored into an USB video card (Dazzle; Taiwan) for offline data analysis. To minimize bias blood flow velocity and brachial artery diameter are evaluated via an edge-detection and wall-tracking software program (CardiovasculareSuit ${ }^{\mathrm{T}}$; Pisa, Italy). From this synchronized brachial artery diameter and blood flow velocity data, blood flow (the product of lumen cross-sectional area and Doppler velocity) is calculated at $30 \mathrm{~Hz}$. Forearm peripheral vascular resistance is determined as mean BP divided by blood flow. FMD is calculated as the percentage change in peak diameter after cuff deflation from baseline. Time to peak diameter is calculated from the point of cuff deflation to peak artery diameter (39). The shear rate is calculated ( $4 \mathrm{x}$ mean blood velocity / artery diameter) as the area under the curve between the point of deflation and maximal dilation (12).

Evaluation of autonomic nervous system activation: ANS activation is evaluated through heart rate variability (HRV) and blood pressure variability (BPV) as described by Atala et al. (40). Data are collected through continuous non-invasive measurements of pressure waves using digital photoplethysmography (Finometer ${ }^{\circledR}$, Finapres Medical System BV, Netherland). A cuff device is worn on the middle finger of the right hand coupled to an analog-to-digital converter (AT / MCA-CODAS, DATAC Instruments Inc., Akron, Ohio, USA). Continuous signal sampling acquisition is obtained at $1,000 \mathrm{~Hz}$ after resting (lying down for 10 minutes) before FMD. The readings are made for 10 minutes before forearm cuff inflation for all volunteers either on the placebo or a1-adrenergic blocker groups.

Data are recorded into BeatsScope ${ }^{\circledR}$ (Smart Medical, Gloucestershire, United Kingdom) and LabChart ${ }^{\circledR}$ (ADInstruments, Sydney, Australia) software programs for data analysis of histograms showing BPV and 
pulse interval series (RR interval) for power spectrum analysis (HRV). All heartbeats are identified through a specialized algorithm using MAT-LAB MT (MATLAB 6.0, Mathworks, USA) with automatic detection of systolic and diastolic pressure wave events. Pulse intervals (PIs) are calculated as the difference between start and end points of the cycle ( $\mathrm{PI}=$ time point 1 - time point 0$)$. For the time-domain analysis of HRV and BPV data, such as spectrum composition (fast Fourier transform), the CardioSeries ${ }^{\circledR}$ software program is used (www.danielpenteado.com/cardioseries). This analysis requires the removal of transient and discrepant elements and interpolation of consecutive fragments of a time series. The following spectral frequencies are evaluated: very low frequency $(V L F, 0.007-0.04 \mathrm{~Hz})$; low frequency $(L F, 0.04-0.15$ $\mathrm{Hz})$; high frequency $(\mathrm{HF}, 0.15-0.4 \mathrm{~Hz})$; and total power (TP, VLF + LF + HF). Spontaneous baroreflex sensitivity (SBE) is inferred by the square root of (LF / HRV m²) / (LF / BPV m²) (40).

Reproducibility of FMD: the reproducibility of FMD measurements by the study evaluator (GW) (who has performed more than 150 assessments) was assessed according to current recommendations $(38,39)$. Eleven healthy individuals ( 6 males and 5 females aged $38.4 \pm 13.9$ years) were tested in the morning ( 9 h) after a 15-minute rest in supine position (38) from October 23 to November 29, 2019. They were evaluated at two time points one hour apart. Reproducibility for each time interval between pairs of values was expressed as the coefficient of variation (CV) of a single measurement, defined as the standard deviation of the difference between paired values divided by the mean and divided by $\sqrt{2}$ ) (38, 41). The mean CV for baseline diameter of healthy volunteers was $1.4 \mathrm{~mm}$ and mean CV for FMD was $11.0 \%$. The recommended $\mathrm{CV}$ values for diameter $(\mathrm{mm})$ are $<2 \%$ and for FMD are $<15 \%(38)$.

The exercise sessions are performed in two afternoon visits $(1 \mathrm{pm})$ in random order (placebo or a1adrenergic blocker). The volunteers are asked to fast over 6 hours before the session. Sessions are held 5 to 7 days apart and include $A E$ on a cycle ergometer; RE; and CE (AE + RE) as described elsewhere (33).

Aerobic exercise protocol: AE session is performed on a horizontal cycle ergometer (Movement BM4500 Pro, São Paulo, Brazil) and consists of warm-up ( 5 minutes) followed by moderate-intensity $A E$ (60\% HR reserve) for 40 minutes. The volunteers are monitored using a heart rate monitor (POLAR ${ }^{T M} R S 800 C X$ RUN, Helsinki, Finland) and a subjective effort scale (Borg scores 6 to 20). BP and HR measures and Borg scores are recorded at the start, every 5 minutes during the session and up to 15 minutes after exercise.

Resistance exercise protocol: RE consists of lower limb exercise (knee extension, knee flexion, leg pressure and plantar flexion exercises) on a guided weight machine (Movement Perform W8; São Paulo, Brazil), 4 sets with 12 repetitions, $60 \%$ of 1-RM ( 2 concentric: 2 eccentric phases controlled by a metronome). There is a 90-second rest between sets and exercises and the session lasts 40 minutes. BP, $\mathrm{HR}$ and Borg scale are recorded at the start and end of set 4 of each exercise as well as up to 15 minutes after exercise. While performing lower limb strength exercises, the volunteers are not be allowed to hold the hand rests, and an adjustable seat belt is used to stabilize the hip during knee extension and knee flexion (33). 
Combined exercise protocol: $\mathrm{CE}$ consists of $\mathrm{AE}$ for 20 minutes + RE for 20 minutes (two sets of each exercise) as described elsewhere. Similarly, BP, HR and Borg scores are recorded at the start and end of $\mathrm{RE}$ as well as at the start, every 5 minutes and up to 15 minutes after $\mathrm{AE}$.

Additional information: the stress exercise test, 1-RM test or exercise session are not started when resting $\mathrm{BP}$ is greater than $160 \mathrm{mmHg}$ (SBP) and/or $105 \mathrm{mmHg}$ (DBP) (42). If BP remains above the recommended levels at a second check the volunteers are referred to the study cardiologist for evaluation. They will be excluded from the study if the cardiologist determines that they cannot participate.

Data management: to ensure data security and data integrity, all research data will be entered and stored in the REDCap platform at the study site overseen by one of the contributors (LFCB). Only the authors of this study will have access to it. Each participant has the right of access to their own research results.

\section{Statistical analysis}

The Shapiro-Wilk is used to test the normality of data. Values are presented as means \pm standard deviations or medians and $95 \%$ confidence intervals ( $95 \% \mathrm{Cls}$ ) according to the distribution of variables. A multivariate analysis of data is performed using the generalized estimation equations (GEE) for repeated measures with Bonferroni's post-hoc. Pearson's and Spearman's tests are used to assess potential correlations between variables according to their distribution. Statistical analyses are carried out using the Statistical Package for Social Sciences (SPSS) 24.0. A level of significance of $<0.05$ is set for all analyses.

\section{Discussion}

Regular aerobic exercise has shown to improve BP (43) and endothelial function (20) in hypertensive patients. Yet there is still scarce evidence on the benefits of resistance training and combined training on $\mathrm{BP}$ and endothelial function in hypertensive patients. However, studies have reported improvements in muscle mass and strength (44), as well as bone density (44) in response to these types of exercise supporting their inclusion in routine training. Thus, new scientific evidence on the relationship between exercise and hypertension is still needed particularly on the effects of exercise on FMD modulation in individuals with impaired endothelial function (45).

Exacerbated ANS activation is characteristic of hypertension (28); this state may compete with endothelium-dependent vasodilation effects and impair vascular response to exercise. This study may help clarify the effects of ANS activation on FMD of the brachial artery in response to different types of exercise in hypertensive individuals. Knowledge of the impact of ANS activation on post-exercise FMD may give some insight on how to improve exercise prescription for hypertension management.

\section{List Of Acronyms}


FMD, flow-mediated dilation; CV, coefficient of variance; ANS, autonomic nervous system; AE, aerobic exercise; $R E$, resistance exercise; $C E$, combined exercise; SNS, sympathetic nervous system; PWV, pulse wave velocity; NO, nitric oxide; ET-1, endothelin-1; FUC, Fundação Universitária de Cardiologia; IC, Instituto de Cardiologia; BMI, body mass index; IPAQ, International Physical Activity Questionnaire; GFR, glomerular filtration rate; LCl, Laboratory of Clinical Investigation; 1-RM, one-repetition maximum; HR, heart rate; $\mathrm{BP}$, blood pressure; $\mathrm{BPV}$, blood pressure variability; $\mathrm{HRV}$, heart rate variability; $\mathrm{PI}$, pulse interval; $V L F$, very low frequency; LF, low frequency; HF, high frequency; TP, total power; $S B E$, spontaneous baroreflex sensitivity; SBP, systolic blood pressure; DBP, diastolic blood pressure; GEE, generalized estimation equations.

\section{Declarations}

Ethics approval and consent to participate: This study following the principles of the Declaration of Helsinki. The research project was approved by the research ethics committee at Institute of Cardiology of Rio Grande do Sul/University Foundation of Cardiology and registered at clinicaltrials.gov (ID: NCT04371757). Also, all volunteer participants must read and sign a free and informed consent (supplementary material) before entering the study.

Consent for publication: Not Applicable.

Competing interests: The authors declare that there is no competing interests.

Funding: There is no financial support for the research, authorship, and/or publication of this article. However, GW receives a post-doctoral grant from Brazil's National Post-Doctoral Fellowship Program (PNPD).

Authors' contributions: GW is the principal investigator and primary responsible for all research procedures. LFCB is responsible for the randomization and for helping FMD and ANS assessments and overseeing data entering and integrity in the REDCap platform. SGN is the cardiologist responsible for medical consultations, evaluations and monitoring. $\mathrm{MCCl}$ and $\mathrm{AML}$ are project supervisors and are contributing to the design, data analysis and interpretation of results as well as critical review of the final manuscript. Also, all authors have read and approved the manuscript.

Acknowledgements: Authors thank to Carla Finger for her English assistance.

\section{References}

1. Gimbrone MA, García-Cardeña G. Endothelial Cell Dysfunction and the Pathobiology of Atherosclerosis. Circ Res. 2016; 118:620-636. DOI: 10.1161/CIRCRESAHA.115.306301. PMID: 26892962.

2. Vanhoutte PM, Shimokawa H, Feletou M, Tang EH. Endothelial dysfunction and vascular disease - a 30th anniversary update. Acta Physiol (Oxf). 2017; 219:22-96. DOI: 10.1111/apha.12646. PMID: 
26706498.

3. Soloviev MA, Kulakova NV, Semiglazova TA, Borodulina EV, Udut VV. Correction of endothelial dysfunction in patients with arterial hypertension. Bull Exp Biol Med. 2011; 151:183-185. PMID: 22238745.

4. Abebe WaMM. Endothelial dysfunction in diabetes: Potential application of circulating markers as advanced diagnostic and prognostic tools. EPMA Journal. 2010; 1:32--45. DOI: 10.1007/s13167-0100012-7. PMID: Abebe2010.

5. Iantorno M, Campia U, Di Daniele N, Nistico S, Forleo GB, Cardillo C, Tesauro M. Obesity, inflammation and endothelial dysfunction. J Biol Regul Homeost Agents. 2014; 28:169-176. PMID: 25001649.

6. Lind L. Lipids and endothelium-dependent vasodilation-a review. Lipids. 2002; 37:1-15. PMID: 11876256.

7. Vykoukal D, Davies MG. Vascular biology of metabolic syndrome. J Vasc Surg. 2011; 54:819-831. DOI: 10.1016/j.jvs.2011.01.003. PMID: 21439758.

8. Donato AJ, Morgan RG, Walker AE, Lesniewski LA. Cellular and molecular biology of aging endothelial cells. J Mol Cell Cardiol. 2015; DOI: 10.1016/j.yjmcc.2015.01.021. PMID: 25655936.

9. Kublickiene K, Fu XD, Svedas E, Landgren BM, Genazzani AR, Simoncini T. Effects in postmenopausal women of estradiol and medroxyprogesterone alone and combined on resistance artery function and endothelial morphology and movement. J Clin Endocrinol Metab. 2008; 93:18741883. DOI: 10.1210/jc.2007-2651. PMID: 18319309.

10. Pahkala K, Heinonen OJ, Simell O, Viikari JS, Rönnemaa T, Niinikoski H, Raitakari OT. Association of physical activity with vascular endothelial function and intima-media thickness. Circulation. 2011; 124:1956-1963. DOI: 10.1161/CIRCULATIONAHA.111.043851. PMID: 21969011.

11. Green DJ, Dawson EA, Groenewoud HM, Jones $\mathrm{H}$, Thijssen DH. Is flow-mediated dilation nitric oxide mediated?: A meta-analysis. Hypertension. 2014; 63:376-382. DOI:

10.1161/HYPERTENSIONAHA.113.02044. PMID: 24277765.

12. Dawson EA, Cable NT, Green DJ, Thijssen DHJ. Do acute effects of exercise on vascular function predict adaptation to training? Eur J Appl Physiol. 2018; 118:523-530. DOI: 10.1007/s00421-0173724-8. PMID: 29234916.

13. Routledge FS, Hinderliter AL, McFetridge-Durdle J, Blumenthal JA, Paine NJ, Sherwood A. Endothelial function in postmenopausal women with nighttime systolic hypertension. Menopause. 2015; DOI: 10.1097/GME.0000000000000405. PMID: 25563797.

14. Mancia G, Fagard R, Narkiewicz K, Redon J, Zanchetti A, Böhm M, Christiaens T, Cifkova R, De Backer G, Dominiczak A, et al. 2013 ESH/ESC guidelines for the management of arterial hypertension: the Task Force for the Management of Arterial Hypertension of the European Society of Hypertension (ESH) and of the European Society of Cardiology (ESC). Eur Heart J. 2013; 34:2159-2219. DOI: 10.1093/eurheartj/eht151. PMID: 23771844. 
15. James PA, Oparil S, Carter BL, Cushman WC, Dennison-Himmelfarb C, Handler J, Lackland DT, LeFevre ML, MacKenzie TD, Ogedegbe O, et al. 2014 evidence-based guideline for the management of high blood pressure in adults: report from the panel members appointed to the Eighth Joint National Committee (JNC 8). JAMA. 2014; 311:507-520. DOI: 10.1001/jama.2013.284427. PMID: 24352797.

16. Martinez DG, Nicolau JC, Lage RL, Toschi-Dias E, de Matos LD, Alves MJ, Trombetta IC, Dias da Silva VJ, Middlekauff HR, Negrão CE, et al. Effects of long-term exercise training on autonomic control in myocardial infarction patients. Hypertension. 2011; 58:1049-1056. DOI:

10.1161/HYPERTENSIONAHA.111.176644. PMID: 22025377.

17. Hansen AH, Nyberg M, Bangsbo J, Saltin B, Hellsten Y. Exercise training alters the balance between vasoactive compounds in skeletal muscle of individuals with essential hypertension. Hypertension. 2011; 58:943-949. DOI: 10.1161/HYPERTENSIONAHA.111.176529. PMID: 21896936.

18. Nyberg M, Mortensen SP, Hellsten Y. Physical activity opposes the age-related increase in skeletal muscle and plasma endothelin-1 levels and normalizes plasma endothelin-1 levels in individuals with essential hypertension. Acta Physiol (Oxf). 2013; 207:524-535. DOI: 10.1111/apha.12048. PMID: 23227981.

19. Ashor AW, Lara J, Siervo M, Celis-Morales C, Oggioni C, Jakovljevic DG, Mathers JC. Exercise modalities and endothelial function: a systematic review and dose-response meta-analysis of randomized controlled trials. Sports Med. 2015; 45:279-296. DOI: 10.1007/s40279-014-0272-9. PMID: 25281334.

20. Pedralli ML, Eibel B, Waclawovsky G, Schaun MI, Nisa-Castro-Neto W, Umpierre D, Pescatello LS, Tanaka H, Lehnen AM. Effects of exercise training on endothelial function in individuals with hypertension: a systematic review with meta-analysis. J Am Soc Hypertens. 2018; 12:e65-e75. DOI: 10.1016/j.jash.2018.09.009. PMID: 30482668.

21. Inaba Y, Chen JA, Bergmann SR. Prediction of future cardiovascular outcomes by flow-mediated vasodilatation of brachial artery: a meta-analysis. Int J Cardiovasc Imaging. 2010; 26:631-640. DOI: 10.1007/s10554-010-9616-1. PMID: 20339920.

22. Morishima T, Tsuchiya Y, lemitsu M, Ochi E. High-intensity resistance exercise with low repetitions maintains endothelial function. Am J Physiol Heart Circ Physiol. 2018; DOI: 10.1152/ajpheart.00281.2018. PMID: 29856651.

23. Waclawovsky G. Tese de doutorado: Efeito agudo do exercício físico aeróbico, resistido e combinado sobre a integridade endotelial em pacientes hipertensos: Instituto de Cardiologia do Rio Grande do Sul/Fundação Universitária de Cardiologia - Programa de Pós-Graduação em Ciências da Saúde.; 2019.

24. Mitchell JH. Abnormal cardiovascular response to exercise in hypertension: contribution of neural factors. Am J Physiol Regul Integr Comp Physiol. 2017; 312:R851-R863. DOI: 10.1152/ajpregu.00042.2017. PMID: 28381455. 
25. Halliwill JR, Taylor JA, Eckberg DL. Impaired sympathetic vascular regulation in humans after acute dynamic exercise. J Physiol. 1996; 495 ( Pt 1):279-288. DOI: 10.1113/jphysiol.1996.sp021592. PMID: 8866370.

26. Atkinson CL, Lewis NC, Carter HH, Thijssen DH, Ainslie PN, Green DJ. Impact of sympathetic nervous system activity on post-exercise flow-mediated dilatation in humans. J Physiol. 2015; 593:51455156. DOI: 10.1113/JP270946. PMID: 26437709.

27. Alves MJ, Rondon MU, Santos AC, Dias RG, Barretto AC, Krieger EM, Middlekauff HR, Negrão CE. Sympathetic nerve activity restrains reflex vasodilatation in heart failure. Clin Auton Res. 2007; 17:364-369. DOI: 10.1007/s10286-007-0448-6. PMID: 18043866.

28. Grassi G, Ram VS. Evidence for a critical role of the sympathetic nervous system in hypertension. J Am Soc Hypertens. 2016; 10:457-466. DOI: 10.1016/j.jash.2016.02.015. PMID: 27052349.

29. Esparza-Ros F, Vaqueiro-Cristóbal R, Marfell-Jones M. International Standards for Anthropometric Assessment. Australia: International Society for the Advancement of Kinanthropometry; 2019.

30. Ghorayeb N, Costa RV, Castro I, Daher DJ, Oliveira Filho JA, Oliveira MA, Cardiologia SBd. [Guidelines on exercise and sports cardiology from the Brazilian Society of Cardiology and the Brazilian Society of Sports Medicine]. Arq Bras Cardiol. 2013; 100:1-41. PMID: 23568146.

31. Levey AS, Stevens LA, Schmid CH, Zhang YL, Castro AF, Feldman HI, Kusek JW, Eggers P, Van Lente F, Greene T, et al. A new equation to estimate glomerular filtration rate. Ann Intern Med. 2009; 150:604612. DOI: 10.7326/0003-4819-150-9-200905050-00006. PMID: 19414839.

32. Brandão AA, Amodeo C, Alcântara C, Barbosa E, Nobre F, Pinto F, Vilela-Martin JF, Bastos JM, YugarToledo JC, Mota-Gomes MA, et al. I Luso-Brazilian Positioning on Central Arterial Pressure. Arq Bras Cardiol. 2017; 108:100-108. DOI: 10.5935/abc.20170011. PMID: 28327876.

33. Waclawovsky G, Umpierre D, Figueira FR, de Lima ES, Alegretti AP, Schneider L, Matte US, Rodrigues TC, Schaan BD. Exercise on Progenitor Cells in Healthy Subjects and Patients with Type 1 Diabetes. Med Sci Sports Exerc. 2015; DOI: 10.1249/MSS.0000000000000764. PMID: 26312614.

34. Torvik D, Madsbu HP. An open one-year comparison of doxazosin and prazosin for mild to moderate essential hypertension. Am J Cardiol. 1987; 59:68G-72G. DOI: 10.1016/0002-9149(87)90160-3. PMID: 2884855.

35. Jones H, Lewis NC, Green DJ, Ainslie PN, Lucas SJ, Tzeng YC, Grant EJ, Atkinson G. a1Adrenoreceptor activity does not explain lower morning endothelial-dependent, flow-mediated dilation in humans. Am J Physiol Regul Integr Comp Physiol. 2011; 300:R1437-1442. DOI: 10.1152/ajpregu.00042.2011. PMID: 21451140.

36. Tymko MM, Tremblay JC, Steinback CD, Moore JP, Hansen AB, Patrician A, Howe CA, Hoiland RL, Green DJ, Ainslie PN. UBC-Nepal Expedition: acute alterations in sympathetic nervous activity do not influence brachial artery endothelial function at sea level and high altitude. J Appl Physiol (1985). 2017; 123:1386-1396. DOI: 10.1152/japplphysiol.00583.2017. PMID: 28860174.

37. Lewis NC, Ainslie PN, Atkinson G, Jones H, Grant EJ, Lucas SJ. Initial orthostatic hypotension and cerebral blood flow regulation: effect of a1-adrenoreceptor activity. Am J Physiol Regul Integr Comp 
Physiol. 2013; 304:R147-154. DOI: 10.1152/ajpregu.00427.2012. PMID: 23174860.

38. Thijssen DHJ, Bruno RM, van Mil ACCM, Holder SM, Faita F, Greyling A, Zock PL, Taddei S, Deanfield $J E$, Luscher T, et al. Expert consensus and evidence-based recommendations for the assessment of flow-mediated dilation in humans. Eur Heart J. 2019; DOI: 10.1093/eurheartj/ehz350. PMID: 31211361.

39. Thijssen DHJ, Black Ma, Pyke KE, Padilla J, Atkinson G, Harris Ra, Parker Ba, Widlansky ME, Tschakovsky ME, Green DJ. Assessment of flow mediated dilation (FMD) in humans: a methodological and technical guideline. American journal of physiology Heart and circulatory physiology. 2010; DOI: 10.1152/ajpheart.00471.2010.

40. Atala MM, Goulart A, Guerra GM, Mostarda C, Rodrigues B, Mello PR, Casarine DE, Irigoyen MC, Pereira AC, Consolim-Colombo FM. Arg16Gly and GIn27Glu $\beta 2$ adrenergic polymorphisms influence cardiac autonomic modulation and baroreflex sensitivity in healthy young Brazilians. Am J Transl Res. 2015; 7:153-161. PMID: 25755837.

41. Donald AE, Halcox JP, Charakida M, Storry C, Wallace SM, Cole TJ, Friberg P, Deanfield JE. Methodological approaches to optimize reproducibility and power in clinical studies of flowmediated dilation. J Am Coll Cardiol. 2008; 51:1959-1964. DOI: 10.1016/j.jacc.2008.02.044. PMID: 18482664.

42. Malachias MVB, Franco RJ, Forjaz CLM, Pierin AMG, Gowdak MM, Klein MRST, Matsudo V. 7th Brazilian Guideline of Arterial Hypertension: Chapter 6 - Non-pharmacological treatment. Arq Bras Cardiol. 2016; 107:30-34. DOI: 10.5935/abc.20160156. PMID: 27819385.

43. Pescatello LS, MacDonald HV, Lamberti L, Johnson BT. Exercise for Hypertension: A Prescription Update Integrating Existing Recommendations with Emerging Research. Curr Hypertens Rep. 2015; 17:87. DOI: 10.1007/s11906-015-0600-y. PMID: 26423529.

44. Garber CE, Blissmer B, Deschenes MR, Franklin BA, Lamonte MJ, Lee IM, Nieman DC, Swain DP, American College of Sports M. American College of Sports Medicine position stand. Quantity and quality of exercise for developing and maintaining cardiorespiratory, musculoskeletal, and neuromotor fitness in apparently healthy adults: guidance for prescribing exercise. Med Sci Sports Exerc. 2011; 43:1334-1359. DOI: 10.1249/MSS.0b013e318213fefb. PMID: 21694556.

45. Nakanishi R, Baskaran L, Gransar H, Budoff MJ, Achenbach S, Al-Mallah M, Cademartiri F, Callister TQ, Chang HJ, Chinnaiyan K, et al. Relationship of Hypertension to Coronary Atherosclerosis and Cardiac Events in Patients With Coronary Computed Tomographic Angiography. Hypertension. 2017; 70:293-299. DOI: 10.1161/HYPERTENSIONAHA.117.09402. PMID: 28607128.

\section{Figures}




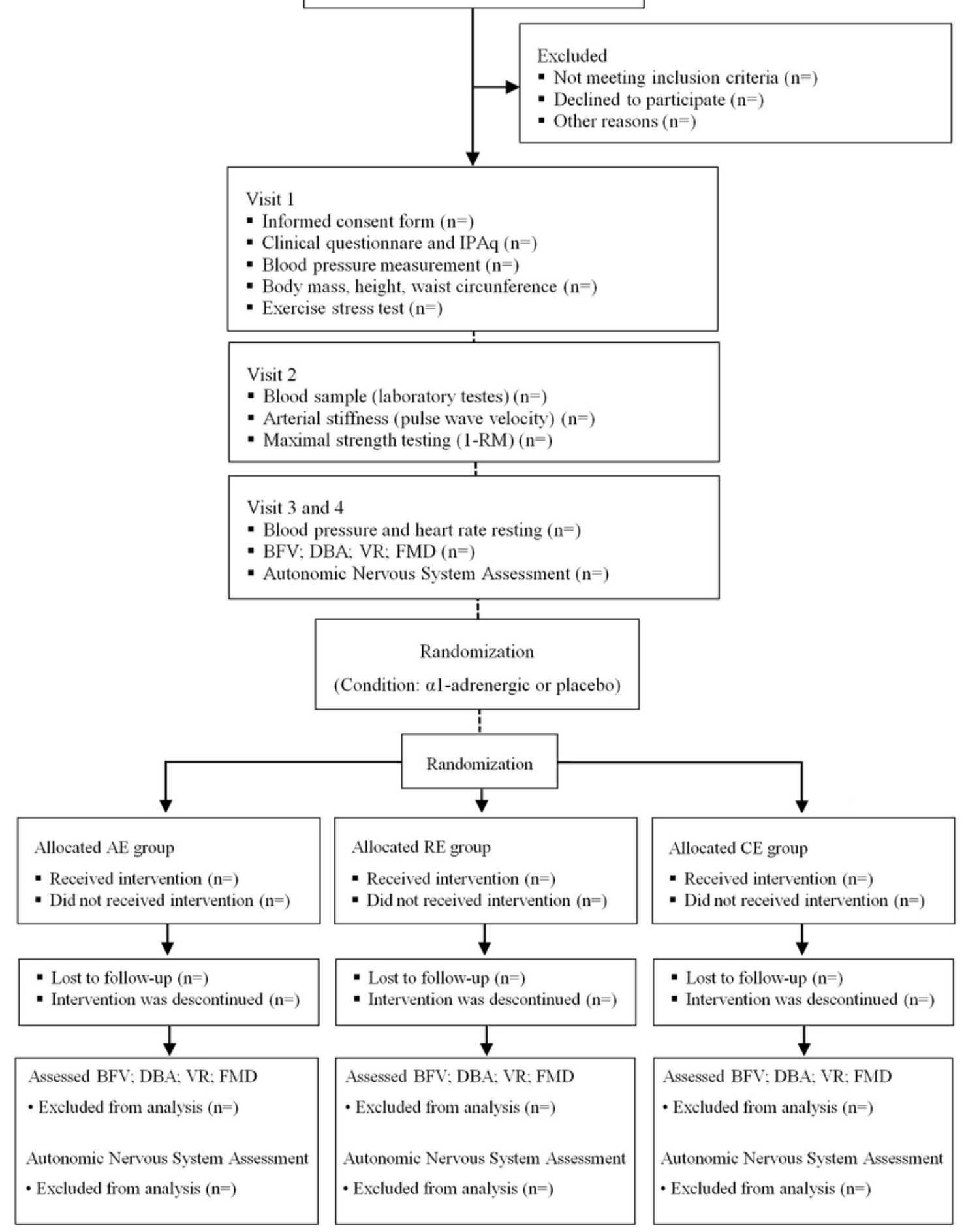

\section{Figure 1}

Study design. IPAq: International Physical Activity Questionnaire; AE: aerobic exercise session; RE: resistance exercise session; $\mathrm{CE}$ : combined exercise; FMD: flow-mediated dilation; BFV: blood flow velocity; DBA: diameter of brachial artery; VR: vascular resistance. 


\begin{tabular}{|c|c|c|c|c|c|c|c|c|c|}
\hline \multirow{3}{*}{ TIMEPOINT } & \multicolumn{9}{|c|}{ STUDY PERIOD } \\
\hline & \multirow{2}{*}{$\begin{array}{c}\text { Enrolment } \\
-t_{1}\end{array}$} & \multirow{2}{*}{$\frac{\text { Enrolment }}{-t_{2}}$} & \multirow{2}{*}{$\begin{array}{c}\text { Enrolment } \\
-t_{3}\end{array}$} & \multirow{2}{*}{$\begin{array}{c}\text { Allocation } \\
0\end{array}$} & \multicolumn{5}{|c|}{ Trial period } \\
\hline & & & & & $t_{1}$ & Exercise & $t_{2}$ & $t_{3}$ & $t_{4}$ \\
\hline \multirow{12}{*}{$\begin{array}{l}\quad \text { ENROLMENT: } \\
\text { Eligibility screen } \\
\text { Informed consent } \\
\text { Clinical questionnare } \\
\text { IPAq } \\
\text { BP measurement } \\
\text { Anthropometry } \\
\text { Exercise stress test } \\
\text { Blood sampimping } \\
\text { Arterial stiffness } \\
\text { 1-RM } \\
\text { Allocation ** }\end{array}$} & $\mathrm{X}$ & $\mathbf{X}$ & $\mathbf{X}$ & & & & & & \\
\hline & $\mathrm{X}$ & & & & & & & & \\
\hline & & $\mathrm{X}$ & & & & & & & \\
\hline & & $x$ & & & & & & & \\
\hline & & $X$ & & & & & & & \\
\hline & & $\mathrm{X}$ & & & & & & & \\
\hline & & $\mathrm{X}$ & & & & & & & \\
\hline & & $x$ & & & & & & & \\
\hline & & & $X$ & & & & & & \\
\hline & & & $x$ & & & & & & \\
\hline & & & $x$ & & & & & & \\
\hline & & & & $\mathrm{X}-\mathrm{X}$ & & & & & \\
\hline \multirow{4}{*}{$\begin{array}{l}\text { INTERVENTIONS } \\
\text { Aerobic } \text { : } \\
\text { Resercistance exercise } \\
\text { Combined exercise }\end{array}$} & & & & & & $X-X$ & & & \\
\hline & & & & & & $X-X$ & & & \\
\hline & & & & & & $X-X$ & & & \\
\hline & & & & & & $X-X$ & & & \\
\hline \multirow[t]{2}{*}{ ASSESSMENTS ${ }^{* *}:$} & & & & & $X-X$ & & & & \\
\hline & & & & & $x-x$ & & & & \\
\hline$D B A$ & & & & & $x-x$ & & & & \\
\hline FMD & & & & & $X-X$ & & - & & \\
\hline ANS Assessment & & & & & $x-x$ & & - & & \\
\hline Blood pressure & & & & & $x-x$ & & 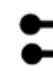 & & 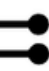 \\
\hline Vascular resistance & & & & & $x-x$ & & $\div$ & & \\
\hline
\end{tabular}

Figure 2

Schedule of enrolment, interventions, and assessment. Adapted from SPIRIT Figure 2013. ${ }^{\star}$ Subjects will be allocated to perform twice $(\mathrm{X}-\mathrm{X})$ each exercise session on alpha-1 adrenergic block and placebo conditions. IPAq: International Physical Activity Questionnaire; BP: blood pressure; 1-RM: one-repetition maximum; BFV: blood flow velocity; DBA: diameter of brachial artery; FMD: flow-mediated dilation; ANS: autonomic nervous system. 


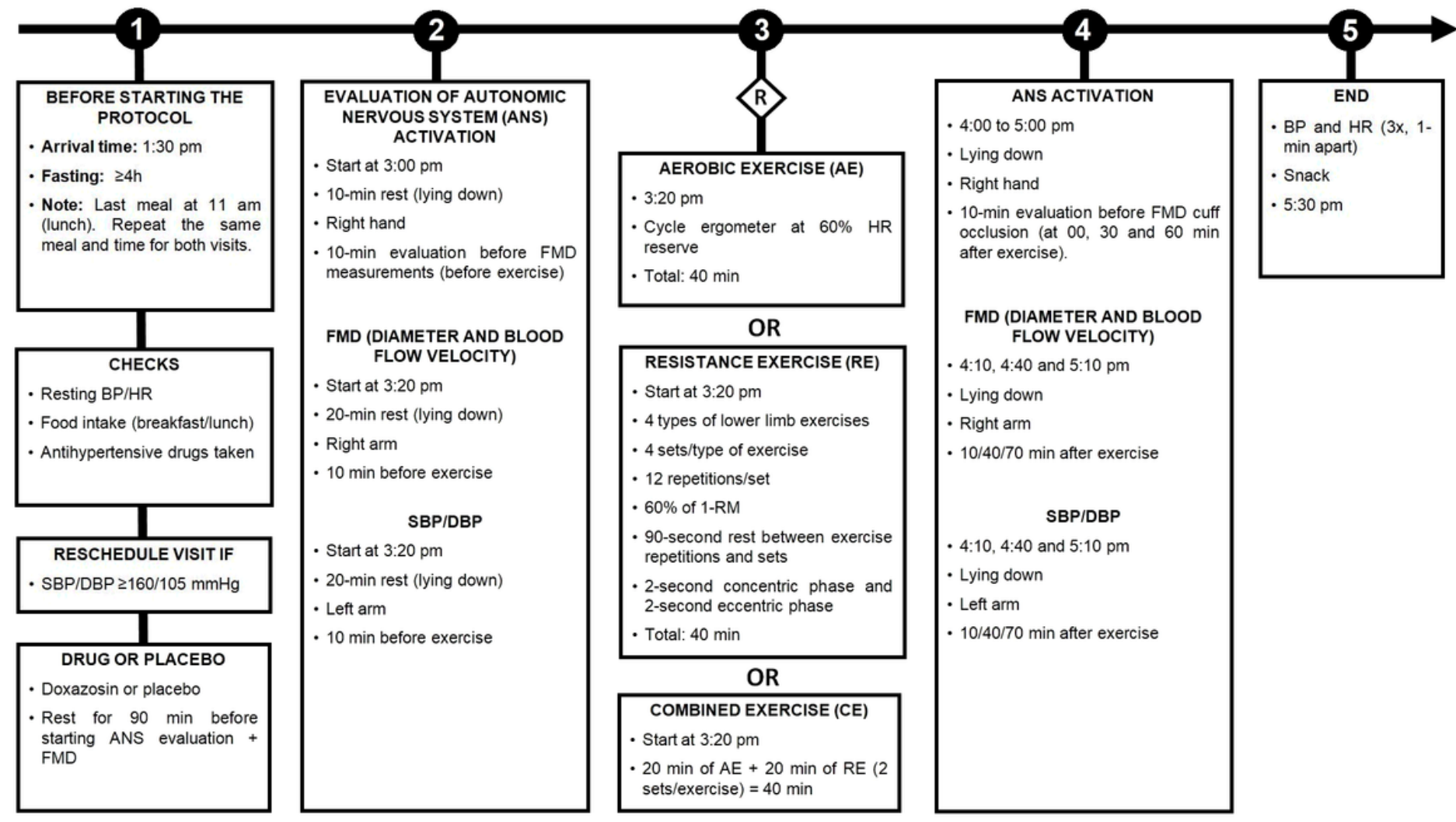

Figure 3

Schedule of intervention day. BP: blood pressure; HR: heart rate; SBP: systolic BP; DBP: diastolic BP; ANS: autonomic nervous system; FMD: flow-mediated dilation; 1-RM: one-repetition maximum.

\section{Supplementary Files}

This is a list of supplementary files associated with this preprint. Click to download.

- Freeandinformedconsentform.doc

- ChecklistSPIRIT.doc 